\title{
A FORMULA FOR THE SOLUTION OF THE NAVIER-STOKES EQUATIONS BASED ON A METHOD OF CHORIN
}

\author{
BY J. MARSDEN ${ }^{1}$
}

Communicated by Murray Protter, July 17, 1973

1. Introduction. Recently $A$. Chorin has found a numerical scheme for solving the Navier-Stokes equations which has the pleasing feature of not breaking down at high Reynolds numbers $R$ [5]. The purpose of this announcement is to present a formula ((2) below) which is designed to establish the convergence of Chorin's time step iteration procedure, assuming that the relevant equations (heat equation and Euler's equations) are solved exactly at each step. Computing the error in the steps involving Euler's equation has been studied by Dushane [6].

For the case of regions with no boundary, the formula has been established by Ebin-Marsden [7] and Marsden [9]. That case is not of direct physical interest since it is shown that as $R \rightarrow \infty$, the solutions converge to the solutions of the Euler equations. This is interpreted as showing a lack of turbulence.

For regions with boundary, where we expect turbulent phenomena, the formula has an interesting new feature due to the lack of compatibility of boundary conditions of the Euler and Navier-Stokes equations. The new term in the formula has the effect of creating vorticity at the boundary.

What we aim to show is that the formula yields, in the limit as the size of the time step $\rightarrow 0$, an exact solution of the Navier-Stokes equations. The formula can actually be used as an existence theorem, and for fixed initial data, yields a time of existence independent of $R$ as $R \rightarrow \infty$. This is a fundamental improvement over known existence theorems for these equations [8]. This should imply the existence of smooth turbulent solutions of the Navier-Stokes equations (see [8], [11]). Moreover, the formula converges as long in time as there is known to be an a priori smooth solution of the Navier-Stokes equations.

AMS (MOS) subject classifications (1970). Primary 35Q10, 76D10; Secondary 58D10, 47D05.

Key words and phrases. Navier-Stokes equations, product formulas, turbulence, boundary layer.

${ }^{1}$ Partially supported by NSF grant GP-15735. 
The last remark can be rephrased by saying that, as long as the formula converges, the limit is an exact solution of the Navier-Stokes equations. Since Chorin's scheme seems to converge experimentally at interestingly large Reynolds numbers, this leads credence to the hypothesis that turbulence is a phenomenon derivable from the Navier-Stokes equations themselves. Most theories of turbulence, in particular that of RuelleTakens [12], rely crucially on this assumption.

We shall present details of the results sketched here in [10]. Despite the intricacy and length of the eventual proof, we endeavor here to sketch the main ideas.

In Chorin [5], the calculations are done in two dimensions, but in our discussion the dimension can be two or three. For concreteness we work on interior domains, but it should be routine to modify the arguments to exterior domains, as it is for many similar problems.

The author thanks A. Chorin for introducing the problem to him and for his encouragement.

2. Statement of the formula. Fix a domain $M$ with smooth boundary $\partial M$. For any vector field $X$ on $M$ we can write uniquely $X=Y+\operatorname{grad} p$ where $\operatorname{div} Y=0$ and $Y$ is parallel to $\partial M$. Set $P(X)=Y$ so $P$ is a projection operator. Let $\Delta$ denote the Laplace operator and set $\tilde{\Delta}=(1 / R)(P \cdot \Delta)$. Define the operator $Z(v)=-P((v \cdot \nabla) v)$.

The Navier-Stokes equations read

$$
\partial v / \partial t=\tilde{\Delta} v+Z(v), \quad v=0 \quad \text { on } \partial M .
$$

Let $H_{t}$ denote the semigroup generated by $\tilde{\Delta}$ (see, e.g. [8]) with zero boundary conditions. Let $E_{t}$ denote the (local in time) evolution operator for the Euler equations with boundary conditions $v \| \partial M$. See [1], [7]. Let $F_{t}$ be the evolution operator for (1); see [8].

Let $\varphi(v)$ be a potential for $v$; e.g., $\varphi(v)=d \Delta^{-1}(v)$ if $M$ is simply connected, so $v=\delta(\varphi(v)) ; \delta$ is the divergence operator on forms.

Let $g_{l}$ be a $C^{\infty}$ function which is zero on a neighborhood of $\partial M$ and is one inside a distance $d(l)$ from $\partial M$, where $d(l) \leqq$ constant $\cdot l^{r}$ and $n=\operatorname{dim} M, r>4$ if $n=3$, and $r>3$ if $n=2$. Basically, $d(\bar{l})$ will be a measure of the thickness of the boundary layer.

Define the operator

$$
\Phi_{l}(v)=\delta\left(g_{l} \cdot \varphi(v)\right) .
$$

Note that $\Phi_{l}(v)$ is zero on $\partial M$ and has the effect of modifying $v$ near $\partial M$ in such a way as to create vorticity near $\partial M$.

The proposed formula for the solution of problem (1) is

$$
F_{t}(v)=\operatorname{limit}_{n \rightarrow \infty}\left(H_{t / n} \circ \Phi_{t / n} \circ E_{t / n}\right)^{n} v
$$


with each side being valid as long as the other side is. It is contended that for suitable smooth $v\left(v \in W^{2, p}(M), v=0\right.$ on $\left.\partial M\right)$, the convergence is strong in $W^{2, p}$ where $p>3$ if $\operatorname{dim} M=3$, and $p>2$ if $\operatorname{dim} M=2$. One has $t$-differentiability in $L_{p}$.

The interpretation of (2) is that one divides the time interval $[0, t]$ into $N$ steps and iterates the procedure: solve Euler's equations $\rightarrow$ create vorticity $\rightarrow$ solve heat equation $\rightarrow$ solve Euler's equation $\rightarrow \cdots$ etc. The error will be $O(1 / N)$, independent of $R$ as $R \rightarrow \infty$.

3. Product formulas for nonlinear semigroups. We now discuss some general formulas of which (2) is a special case.

Recall that if $A$ and $B$ are two operators satisfying suitable conditions then the Lie-Trotter formula holds:

$$
e^{t(A+B)}=\operatorname{limit}_{n \rightarrow \infty}\left(e^{t A / n} \circ e^{t B / n}\right)^{n} .
$$

Chernoff [3] has generalized this as follows. Let $K(t)$ be a family of operators and let $A=d K(t) /\left.d t\right|_{t=0}$. Then under suitable conditions, the semigroup $F_{t}$ generated by $A$ is given by

$$
F_{t}=\operatorname{limit}_{n \rightarrow \infty} K(t / n)^{n} .
$$

One recovers (3) using $K(t)=e^{t A} \circ e^{t B}$. Formula (4) has been generalized to nonlinear semigroups by Brezis and Pazy [2]. A form suitable for present purposes is found in Marsden [9].

For example, (4) immediately yields the useful formula

$$
e^{\tilde{\Delta} t}=\operatorname{limit}_{n \rightarrow \infty}\left(P e^{t \Delta / n}\right)^{n} .
$$

Similarly, using Lagrangian coordinates, it is easy to prove

$$
E_{t}=\operatorname{limit}_{n \rightarrow \infty}\left(P \stackrel{\circ}{E}_{t / n}\right)^{n}
$$

where $\stackrel{\circ}{E}_{t}$ denotes the evolution operator for the equation $\partial v / \partial t+(v \cdot \nabla) v=0$ which is known explicitly (see [11]). Actually passing to Lagrangian coordinates and using the methods of [7] seems to allow for simplifications in the proof of formulas like (6), cf. Chorin [4].

We propose that the same method will allow for the proof of (2) with

$$
K(t)=H_{t} \circ \Phi_{t} \circ E_{t} .
$$

Although one should use Lagrangian coordinates for the proof, we do not have space to discuss that aspect here. Suffice it to say that in a suitable 
metric $d$, the properties one needs on $K(t)$ to ensure convergence in (2) are (locally):

$$
\begin{aligned}
d(K(t) x, K(t) y) & \leqq e^{\beta t} d(x, y), \\
d(K(t+s) x, K(t) K(s) x) & \leqq \text { (constant)ts. }
\end{aligned}
$$

(See Marsden [9] for techniques for verifying (8). The verification relies on a detailed study of various smoothness properties of the evolution operators $H_{t}$ and $E_{t}$ obtained in [10].)

Actually, what is more interesting is to see why it is that

$$
K^{\prime}(0)=\tilde{\Delta}+Z \text {. }
$$

It seems mysterious at first that $\Phi_{t}$ in (7) does not affect the generator $K^{\prime}(0)$. We shall confine ourselves to discussing this point in $\S 4$.

4. Calculation of the generator. Probably the most crucial aspect of formula (2) is formula (9) where $K(t)$ is given by (7).

In order to see this, write

$$
\begin{aligned}
t^{-1}\left\{H_{t} \Phi_{t} E_{t} v-v\right\}=t^{-1}\{ & {\left[H_{t} \Phi_{t} E_{t} v-H_{t} \Phi_{t} v\right] } \\
& \left.+\left[H_{t} \Phi_{t} v-H_{t} v\right]+\left[H_{t} v-v\right]\right\}
\end{aligned}
$$

The first and last terms in (10) converge, respectively to $Z(v)$ and $\tilde{\Delta} v$ (one needs to know $H_{t} \Phi_{t}$ is $t$-continuous for this). Thus the validity of (9) is assured by the following key lemma:

LEMMA. If $v$ is suitably smooth, $v=0$ on $\partial M$, then in $L_{p}$,

$$
\operatorname{limit}_{t \rightarrow 0} \frac{1}{t}\left[H_{t} \Phi_{t} v-H_{t} v\right]=0 .
$$

Indeed, if $K(t, x, y)$ is a Green's function for $\tilde{\Delta}$ on $M$ then

$$
\begin{aligned}
\frac{1}{t}\left(H_{t} \Phi_{t} v-H_{t} v\right)(x) & =\frac{1}{t} \int_{M} K(t, x, y)\left[\left(\Phi_{t} v\right)(y)-v(y)\right] d y \\
& =\int_{M} \frac{1}{t} d K(t, x, y)\left[g_{t} \varphi(v)(y)-\varphi(v)(y)\right] d y \\
& =\int_{B_{t}} \frac{1}{t} d K(t, x, y)\left[g_{t} \varphi(v) y-\varphi(v)(y)\right] d y
\end{aligned}
$$

where $t=l$ in $g_{l}$ and $d(l)$ above and $B_{t}=\{x \in M \mid d(x, \partial M) \leqq d(t)\}$. Taking into account the nature of the singularity of $K$ and the choice of $d(t)$, we see that in $L_{p}$ norm, the above is majorized by

$$
C_{t} \times \sup _{M}\left[g_{t} \varphi(v)-\varphi(v)\right]
$$


where $C_{t}$, the $L_{p}$ norm of $t^{-1} \int_{B_{t}} d K(t, x, y) d y$ goes to zero as $t \rightarrow 0$ on account of the rapidity with which the volume of $B_{t}$ goes to zero as $t \rightarrow 0$. Thus (11) and hence (9) follows.

\section{REFERENCES}

1. J. P. Bourguignon and H. Brezis, Remarks on the Euler equation (preprint).

2. H. Brezis and A. Pazy, Convergence and approximation of semigroups of nonlinear operators in Banach spaces, J. Functional Analysis 9 (1972), 63-74. MR 45 \#2529.

3. P. R. Chernoff, Note on product formulas for operator semigroups, J. Functional Analysis 2 (1968), 238-242. MR 37 \#6793.

4. A. J. Chorin, On the convergence of discrete approximations to the Navier-Stokes equations, Math. Comp. 23 (1969), 341-353. MR 39 \#3724.

5. — , Numerical study of slightly viscous flows, J. Fluid Mech. 57 (1973), 785796.

6. T. E. Dushane, Convergence of a vortex method for solving Euler's equation, Math. Comp. 27 (1973), 719-728.

7. D. G. Ebin and J. E. Marsden, Groups of diffeomorphisms and the motion of an incompressible fluid, Ann. of Math. (2) 92 (1970), 102-163. MR 42 \#6865.

8. O. A. Ladyženskaja, The mathematical theory of viscous incompressible flow, 2nd rev. ed., Nauka, Moscow, 1970; English transl., Gordon and Breach, New York, 1969. MR 42 \#6442; 40 \#7610.

9. J. E. Marsden, On product formulas for nonlinear semigroups, J. Functional Analysis 13 (1973), 51-74.

10. - Some fundamental properties of the solutions of the Euler and NavierStokes equations (in preparation).

11. J. Marsden, D. Ebin and A. Fischer, Diffeomorphism groups, hydrodynamics and relativity, Proc. 13th biennial seminar of Canadian Math. Congress, ed. J. R. Vanstone, Montreal, Canada (1972), 135-279.

12. D. Ruelle and F. Takens, On the nature of turbulence, Comm. Math. Phys. 20 (1971), 167-192; ibid 23 (1971), 343-344. MR 44 \#1297; 45 \#1478.

Department of Mathematics, University of California, Berkeley, California 94720 